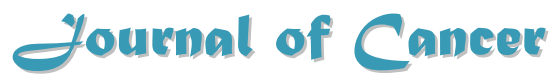

2010; 1:141-149

Research Paper

(C) Ivyspring International Publisher. All rights reserved

\title{
Mesothelin as a Potential Therapeutic Target in Human Cholangiocarci-
}

\section{noma}

\section{Liping $\mathrm{Yu}^{* 1,2}$, Mingqian Feng ${ }^{* 1}$, Heungnam Kim${ }^{1}$, Yen Phung ${ }^{1}$, David E. Kleiner ${ }^{3}$, Gregory J. Gores ${ }^{4}$, Min Qian², Xin Wei Wang ${ }^{5}$, Mitchell Ho1 ${ }^{1}$}

1. Laboratory of Molecular Biology, Center for Cancer Research, National Cancer Institute, National Institutes of Health, Bethesda, Maryland, USA

2. College of Life Sciences, East China Normal University, Shanghai, China

3. Laboratory of Pathology, National Cancer Institute, National Institutes of Health, Bethesda, Maryland, USA

4. Division of Gastroenterology and Hepatology, Mayo Clinic, Rochester, Minnesota, USA

5. Laboratory of Human Carcinogenesis, National Cancer Institute, National Institutes of Health, Bethesda, Maryland USA

*These authors contributed equally to this paper.

Corresponding author: Mitchell Ho, Laboratory of Molecular Biology, Center for Cancer Research, National Cancer Institute, National Institutes of Health, 37 Convent Drive, Room 5002C, Bethesda, MD 20892-4264. Phone: (301) 451-8727; Fax: (301) 402-1344; E-mail: homi@mail.nih.gov

Received: 2010.09.16; Accepted: 2010.10.01; Published: 2010.10.01

\begin{abstract}
Background: Hepatocellular carcinoma (HCC) and cholangiocarcinoma (CCA) are the two most common primary liver cancers, yet there have been no significant advances in effective therapeutics. Mesothelin has been reported as a new therapeutic target in various types of cancer. Here, we investigated the expression of mesothelin in liver cancer and its potential role as a novel therapeutic target for immunotherapy.

Methods: HCC and CCA specimens were examined by immunohistochemistry for mesothelin expression. Protein expression was assessed by immunoblotting and flow cytometry. The SSIP immunotoxin targeting mesothelin was evaluated in the well-established CCA cell lines $\mathrm{HuCCTI}, \mathrm{HuH}-28, \mathrm{KMBC}, \mathrm{KMCH}, \mathrm{Mz}-\mathrm{ChA}-\mathrm{I}$ and OZ.

Results: We showed strong immunochemical mesothelin staining in $33 \%$ of the surgically resected CCA specimens and 3 of 6 CCA cell lines (OZ, KMBC and KMCH). No mesothelin staining was found in $\mathrm{HCC}$ or normal liver tissue. Mesothelin was primarily localized to the cellular plasma membrane and the mature form (molecular weight, $\sim 40 \mathrm{kDa}$ ) was expressed at a high level in CCA tissues. Moreover, 22\% of CCA specimens had a high mesothelin expression level which was comparable to the CCA cell line models. Interestingly, SSIP showed very high and specific growth inhibition when added to mesothelin-expressing CCA cells with $\mathrm{IC}_{50}$ values ranging from 0.5 to $\mathrm{II} \mathrm{ng} / \mathrm{mL}$.

Conclusions: Mesothelin is overexpressed in one-third of CCA tissues. SSIP targeting mesothelin reveals a remarkable single agent activity against CCA in vitro. These findings indicate a potential for SSIP in the immunotherapeutic treatment of CCA.
\end{abstract}

Key words: cholangiocarcinoma, hepatocellular carcinoma, bile duct carcinoma, immunotoxin, mesothelin, SS1P

\section{Introduction}

Liver cancer is one of the most common malignancies worldwide [1]. Hepatocellular carcinoma
(HCC) and cholangiocarcinoma (CCA, or bile duct carcinoma) are the two most common liver cancers in 
humans. Of the several types of cancer originating in the liver, HCC is the most common form. CCA accounts for an estimated $15 \%$ of primary liver cancer worldwide [2]. CCA is a term used broadly to describe adenocarcinomas arising in both the intrahepatic and extrahepatic biliary tract. Intrahepatic CCA is a primary liver cancer that exhibits characteristics of cholangiocyte differentiation [3]. The incidence and mortality rates of CCA have been increasing in Western countries over the past two to three decades $[2,4,5]$. In the United States, intrahepatic CCA accounts for 2,500 new cases of liver cancer a year [6,7]. The etiology of most CCAs remains undetermined. CCA is responsible for more than $60 \%$ of liver tumors in parts of Southeast Asia, especially in northeastern Thailand. Its geographic distribution worldwide coincides with endemic areas of liver flukes [2]. However, there are a number of important risk factors associated with CCA in addition to liver flukes. These include hepatolithiasis, primary sclerosing cholangitis, and cholodochal cysts [2,8]. Although the major etiologies and risk factors for the development of liver cancer have been studied for decades, approximately $90 \%$ of CCA in Western countries develop in the absence of any known etiology. There have been no significant advances in effective therapeutics. Therefore, identifying targeted therapy represents a new therapeutic approach, but remains a challenge partly due to a lack of a good therapeutic target. Thus, the identification of novel therapeutic targets in liver cancer is of paramount importance.

Mesothelin is a tumor differentiation antigen present at low levels on a restricted set of normal adult tissues (e.g. mesothelium), and is expressed at high levels in mesotheliomas, as well as ovarian, pancreatic, and lung cancers [9-11]. The mesothelin gene encodes a $71-\mathrm{kDa}$ precursor protein that is processed to a 40-kDa glycosyl-phosphatidylinositol (GPI) -anchored protein, the mature mesothelin [12], present on the cell surface.

The biological functions of mesothelin are undefined. A recent study demonstrates that mesothelin mediates cell adhesion through an interaction with CA125 [13-15], suggesting that there may be an important role for CA125 and mesothelin in the metastatic spread of cancer in the peritoneal cavity. Serum CA125 has also been evaluated as a diagnostic biomarker for primary liver cancer $[16,17]$.

Shed mesothelin has been evaluated as a diagnostic serum biomarker for ovarian cancer and mesothelioma [18-22]. Upon evaluation of the immunological response to mesothelin-expressing tumors, we previously describe that the antibodies specific for mesothelin are elevated in the sera of patients with mesothelioma and epithelial ovarian cancer [23].

Mesothelin has also been suggested as a therapeutic target in mesothelioma, ovarian, and pancreatic cancers [24]. An anti-mesothelin recombinant immunotoxin, SS1(dsFv)PE38, or SS1P, composed of the Fv fragment of the antibody SS1 and a truncated form of Pseudomonas exotoxin A (PE), has been evaluated in Phase I studies [24,25]. Upon binding to mesothelin, the immunotoxin is internalized, undergoes processing in the endocytic compartment and the immunotoxin fragment containing the ADP-ribosylation domain is transported to the endoplasmic reticulum. It is then translocated to the cytosol where it inhibits elongation factor-2 leading to inhibition of protein synthesis and ultimately cell death. A mouse-human chimeric IgG monoclonal antibody (mAb) based on the SS1 Fv has been developed and is currently being evaluated in a Phase I clinical study enrolling patients with mesothelin-positive pancreatic, mesothelioma, non-small cell lung and ovarian cancers [26].

Immunotoxin SS1P, in which the Fv was obtained from an antibody phage library, binds the $\mathrm{N}$ terminal (Region I) of cell surface-bound mesothelin [15]. Mouse mAbs, MN and MB, were generated in mesothelin-deficient mice by DNA immunizations followed by a single boost of a recombinant mesothelin-Fc fusion protein [27]. Both $\mathrm{MN}$ and immunotoxin SS1P bind to Region I, which is the most immunogenic in mesothelin. However, $\mathrm{MB}$ reacts with an unknown epitope and its epitope does not overlap the $\mathrm{MN}$ binding site. The $5 \mathrm{~B} 2 \mathrm{mAb}$ was generated by immunizing mice with a recombinant prokaryotic fusion protein corresponding to 100 amino acids which is also present in the $\mathrm{N}$ terminal Region I of mesothelin. Even though both $\mathrm{MB}$ and 5B2 work for immunohistochemistry (IHC) [11], 5B2 reacts with the bacterial form of mesothelin, but MB does not, indicating the binding of $\mathrm{MB}$ to mesothelin may be glycosylation dependent.

In spite of the recent evidence showing mesothelin expression in various solid tumors, mesothelin has yet to be thoroughly investigated in primary liver cancer. Here we characterized the mesothelin expression in liver cancer by IHC, Western blotting and flow cytometry, and investigated mesothelin as a potential therapeutic target using the SS1P immunotoxin. We demonstrated that SS1P exhibited remarkably high and specific growth inhibition against mesothelin-expressing CCA cells, and should be evaluated as a novel therapeutic agent for the immunotherapy of CCA. 


\section{Materials and Methods}

\section{Tumor samples}

Frozen and fixed liver tumor samples were acquired from the Cooperative Human Tissue Network (Charlottesville, VA). A set of tissue microarray slides containing samples of normal and neoplastic liver tissue were obtained from Pantomics (Richmond, CA).

We followed the REMARK guidelines [28] to analyze tumor samples. A total of 87 tissue samples were analyzed in this study as follows: 10 normal liver tissues, $63 \mathrm{HCC}$ and 14 CCA. The patients' age at diagnosis varied from 18 to 70 years (mean 47 yr; median $47 \mathrm{yr}$ ). The tumors were sampled from patients at stage I $(14 \%)$, stage I-II (16\%), stage II (36\%), stage II-III $(16 \%)$, and stage III (5\%), whereas $13 \%$ of the tumors were at an unknown stage. Duplicate tissue specimens were analyzed for each patient.

\section{Cell lines}

A panel of six human HCC cell lines was obtained from the National Cancer Institute (NCI) Laboratory of Human Carcinogenesis, Bethesda, Maryland. They include SK-Hep1, HepG2, Hep3B, Huh-1, Huh-4, and Huh-7. A panel of the six human CCA lines (HuCCT1, OZ, Mz-ChA-1, KMBC, KMCH, and $\mathrm{HuH}-28$ ) was obtained from Dr. Gregory J. Gores of the Mayo Clinic, Rochester, Minnesota. OVCAR-3 (human epithelial ovarian cancer cell line) was obtained from American Type Culture Collection (ATCC; Manassas, VA). H9 is a transfected A431 human epithelial carcinoma cell line that stably expresses human mesothelin [23]. The cell lines were cultured in RPMI or DMEM supplemented with $10 \%$ fetal bovine serum, $100 \mathrm{U} / \mathrm{mL}$ penicillin, $0.1 \mathrm{mg} / \mathrm{mL}$ streptomycin, and $2 \mathrm{mmol} / \mathrm{L} \mathrm{L-glutamine.} \mathrm{In} \mathrm{addi-}$ tion, recombinant human insulin $(10 \mu \mathrm{g} / \mathrm{ml})$ (Eli Lilly, Indianapolis, IN) was added to all of the cultures of the OVCAR-3 cell line. G418 $(700 \mu \mathrm{g} / \mathrm{ml})$ was added to all of the cultures of the $\mathrm{H} 9$ cell line.

\section{Immunoblot analysis}

RIPA buffer ( $25 \mathrm{mmol} / \mathrm{L}$ Tris-HCl (pH 7.6), 150 $\mathrm{mmol} / \mathrm{L} \mathrm{NaCl}, 1 \% \mathrm{NP}-40,1 \%$ sodium deoxycholate, $0.1 \%$ SDS) containing $2 \%$ SDS and protease inhibitor cocktail tablets (Roche Applied Science, Indianapolis, IN) was used to solubilize cells on tissue culture dishes. Protein concentrations were determined by the bicinchoninic acid protein assay (Pierce, Rockford, IL) according to the manufacturer's protocol. Equivalent amounts (40 $\mu \mathrm{g}$ per lane) of whole-cell lysates were separated by a $4 \%$ to $20 \%$ Tris-glycine SDS-PAGE gradient gel and subsequently transferred onto nitrocellulose membranes. A lesser amount $(2 \mu \mathrm{g}$ per lane) of positive (H9) and negative (A431) control cell lysates were loaded onto the SDS-PAGE gradient gel due to very high mesothelin signals in Western blots, which were done following the established protocol [11]. Each blot was treated with $1 \mu \mathrm{g} / \mathrm{mL}$ of anti-mesothelin mAb MN (Rockland, Gilbertsville, PA) [27] or a new rabbit mAb specific for mesothelin (MH and YP, unpublished data). Primary antibodies were detected by secondary goat anti-mouse or anti-rabbit antibodies conjugated with horseradish peroxidase (Jackson ImmunoResearch Laboratories, West Grove, PA). Signals were visualized by using the Enhanced Chemiluminescence Kit (GE Healthcare, Piscataway, NJ).

\section{Flow cytometry}

Flow cytometric analysis was used to measure the antigen expression on cancer cells. In a typical protocol for flow cytometric analysis [11], $5 \times 10^{5}$ cells were incubated with $2 \mu \mathrm{g} / \mathrm{mL}$ of anti-mesothelin $\mathrm{mAb} \mathrm{MN}$ diluted with $500 \mu \mathrm{L}$ of PBS containing $5 \%$ bovine serum albumin and $0.02 \%$ sodium azide. After incubation for 1 hour at $4^{\circ} \mathrm{C}$, the cells were washed once with PBS and incubated with a 1:200 dilution of Goat $\mathrm{F}\left(\mathrm{ab}^{\prime}\right)_{2}$ Anti-Mouse Ig's, R-PE Conjugate (Invitrogen/BioSource, Carlsbad, CA) for 1 hour. After washing twice with PBS, the cells were suspended in $0.5 \mathrm{~mL} \mathrm{PBS}$, and the fluorescence associated with the live cells was measured using a FACSCalibur flow cytometer (BD Biosciences, San Jose, CA).

\section{Immunohistochemistry}

IHC was conducted following previously established protocols $[11,21,27,29]$. In brief, after initial deparaffinization/hydration, antigen retrieval was performed by microwaving in $10 \mathrm{mM}$ citrate buffer, $\mathrm{pH} 6.0$ for 5 minutes at maximum power and then transferring to a pre-warmed steamer for 15 minutes to unmask the epitopes. The slides were incubated overnight at $4^{\circ} \mathrm{C}$ with a primary mouse $\mathrm{mAb}$ against mesothelin: 5B2 (Novocastra, Newcastle-upon-Tyne, UK, clone; 1:40 dilution) or MB (1 $\mu \mathrm{g} / \mathrm{mL})$ (Rockland) [27]. Detection was done using the avidin-biotinylated peroxidase method with 3,3'-diaminobenzidine tetrahydrochloride (ABC Kit, Vector, Burlingame, CA). Finally, tissues were counterstained with hematoxylin, dehydrated, and coverslips were applied. Cell staining was examined on a Zeiss Axio Observer Z1 microscope (Carl Zeiss MicroImaging, Inc. Thornwood, NY), and images were collected with Axiovision software (Carl Zeiss MicroImaging, Inc.).

Mesothelin expression was characterized following previously established procedures $[11,21,27,29]$. Mesothelin was detected by staining of 
the surface of tumor cells and the staining graded as positive if at least $30 \%$ of accessible tumor cells were labeled. This cutoff was based on the requirement for the clinical trial of the anti-mesothelin immunotoxin, SS1P, for which these patients were being screened. Similar cutoffs for antigen expression have been used for other immunotoxin studies $[11,21,27,29]$. However, it is possible that patients who were defined as having mesothelin-negative tumors could have mesothelin expression in a small percentage of tumor cells.

\section{Cell proliferation assays}

The growth inhibition of immunotoxin SS1P on mesothelin-expressing liver cancer cells was determined on liver cancer cell lines by a WST- 8 cell proliferation assay as described previously [11]. Cancer cells $(10,000$ per well) in 96-well tissue culture plates were incubated with various concentrations of SS1P for 72 hours because all the cancer cell lines examined
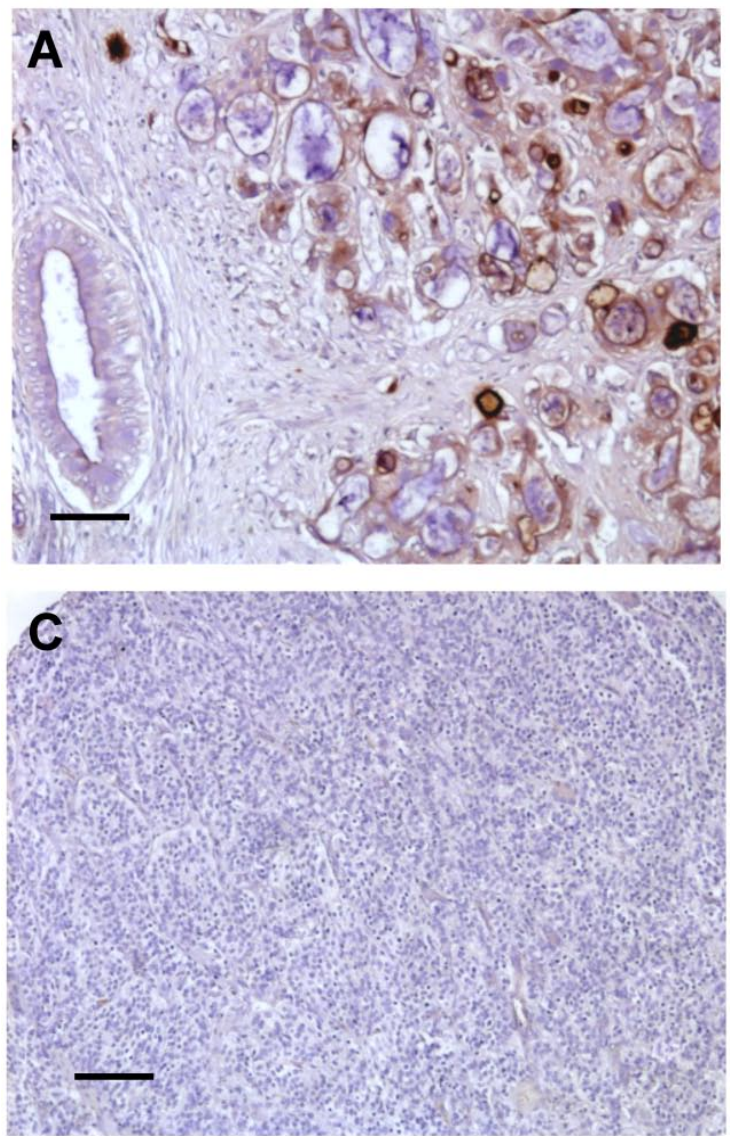

in this study reached optimal population densities within 48 to 72 hours.

\section{Results}

\section{Mesothelin expression in human liver cancers}

To examine the expression of mesothelin and its cellular localization in liver cancer, we examined human liver cancer specimens by IHC using the 5B2 $\mathrm{mAb}$ following established protocols $[11,21,27,29]$. IHC staining of CCA showed mainly staining of the cell membrane with weaker cytoplasmic staining (Fig. 1). The intensity of staining varied from cell to cell and from tumor to tumor but all CCA tumors tested showed staining of more than $30 \%$ of the tumor cells (Fig. 1A and 1B). The desmoplastic stroma around the invasive tumor did not stain. Some staining of the apical brush border of large bile ducts was also seen. In contrast, mesothelin was not detected in both HCC and normal liver tissue (Fig. 1C and 1D). Each immunoreactivity pattern was confirmed by duplicate specimens from the same patient.
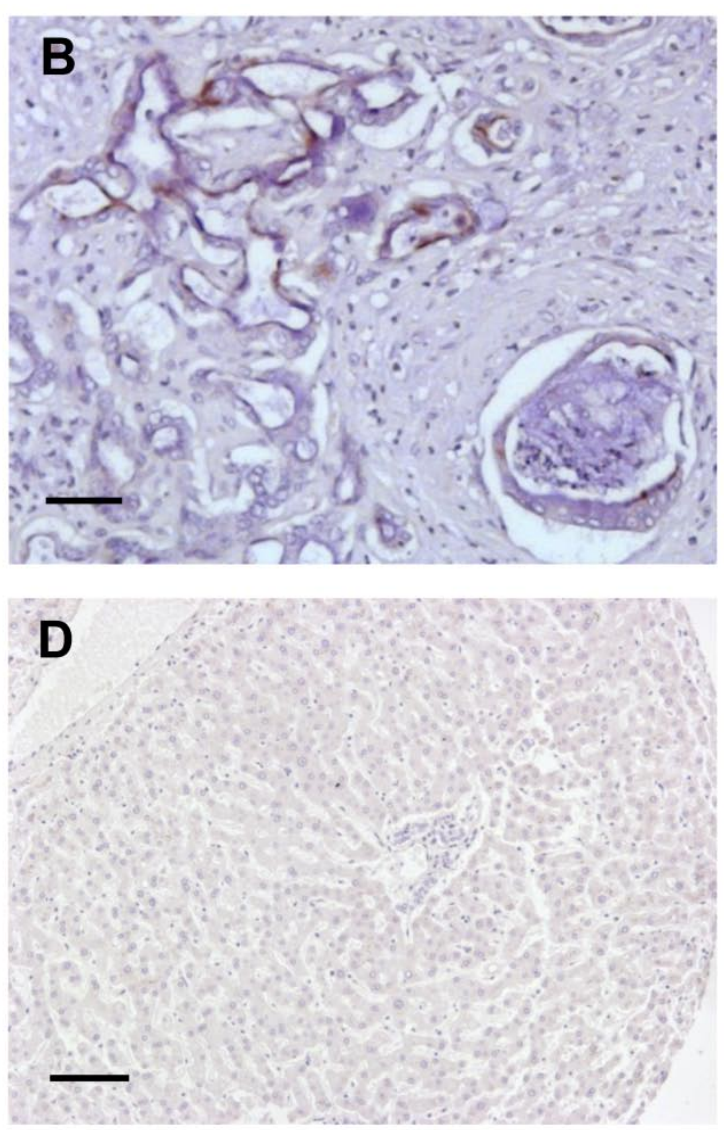

Figure I. Summary of immunoreactivity patterns in cancer patient specimens. Immunohistochemical evaluation using the 5B2 mAb showed high (A) and low (B) expression of mesothelin in two representative CCA specimens. No staining was found in both HCC (C) and normal liver tissues (D). Each immunoreactvity pattern was confirmed by duplicate specimens from the same patient. Scale bar, $100 \mu \mathrm{m}$. 


\section{Characterization of mesothelin protein expres- sion}

The mesothelin molecules in liver tumors have not been characterized by immunoblotting. Here we analyzed the protein expression of mesothelin in 12 liver cancer cell lines and 18 tumor specimens by immunoblotting using the MN mAb. We examined a panel of 12 human liver cancer cell lines: 6 CCA (HuCCT1, OZ, Mz-ChA-1, KMBC, KMCH, and HuH-28) and 6 HCC (Huh-1, Huh-4, Huh-7, HepG2, Hep3B and SK-Hep-1). In Figure 2A, the mesothelin mature form (molecular weight, $\sim 40 \mathrm{kDa}$ ) was detected at a high level in 50\% (3 of 6) CCA cell lines $(\mathrm{OZ}, \mathrm{KMBC}$ and $\mathrm{KMCH})$. Mesothelin expression was not detected in the HCC cell lines except for weak mesothelin expression found in SK-Hep-1. A recent study indicated that SK-HEP-1 was not a HCC cell line because it did not have properties of hepatocytes and was endothelial in origin [30].

To compare mesothelin levels in CCA with the CCA cell line models, we obtained a panel of nine CCA and nine HCC specimens from major cancer centers in the USA through the Cooperative Human
Tissue Network. As shown in Figure 2B, mature mesothelin was detected in 33\% (3 of 9) of CCA specimens; $22 \%$ (2 of 9) of CCA specimens had a high mesothelin expression level which is comparable to the CCA cell line models (OZ, KMBC and $\mathrm{KMCH})$, and to mesothelioma, ovarian cancer and lung adenocarcinoma $[11,25]$. Interestingly, one CCA specimen (No. 333) had an extremely high mesothelin expression level with a smear of high molecular weight staining, indicating that the mesothelin molecule in this particular CCA specimen was heavily glycosylated. Mesothelin expression was only weakly detected in one HCC specimen (No. 610) and its expression level was not comparable to the high expression found in CCA specimens (No. 333 and 334). In contrast to our previous observations in lung adenocarcinoma [11], the precursor form of mesothelin (molecular weight, $71-\mathrm{kDa}$, as shown as an upper band in the $\mathrm{H} 9$ control) was not detectable in any of the liver cancer specimens. We decreased the loading of CCA specimen 333 with 5-, 10-, 20-folds less amount of protein and found the major band was about 40-kDa (data not shown).
A

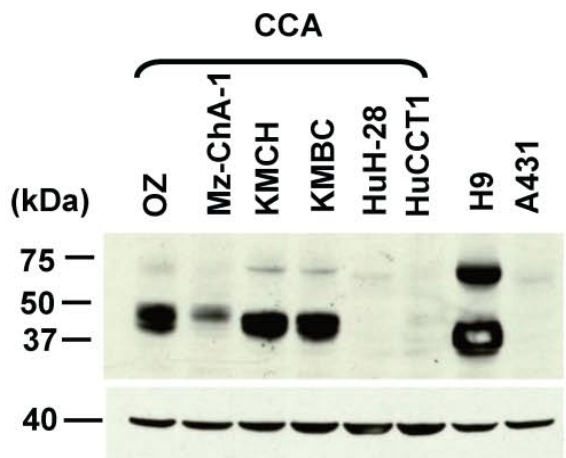

B

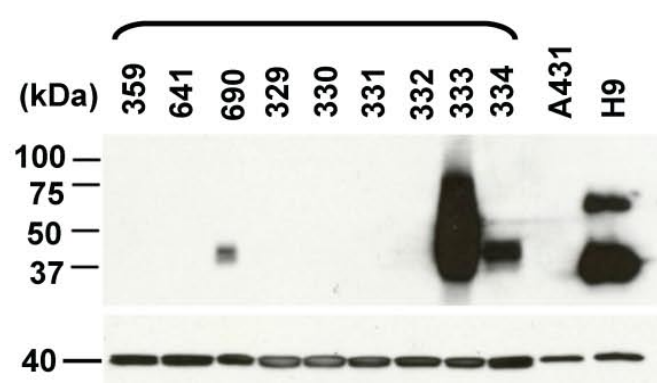

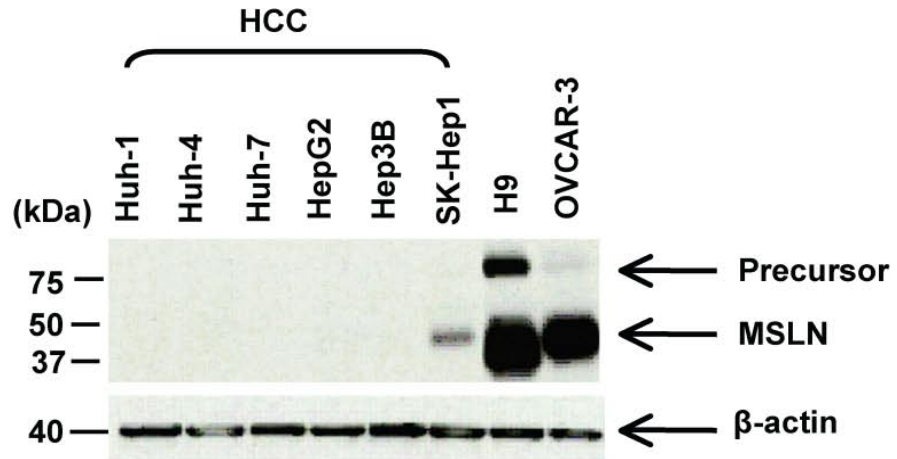

HCC

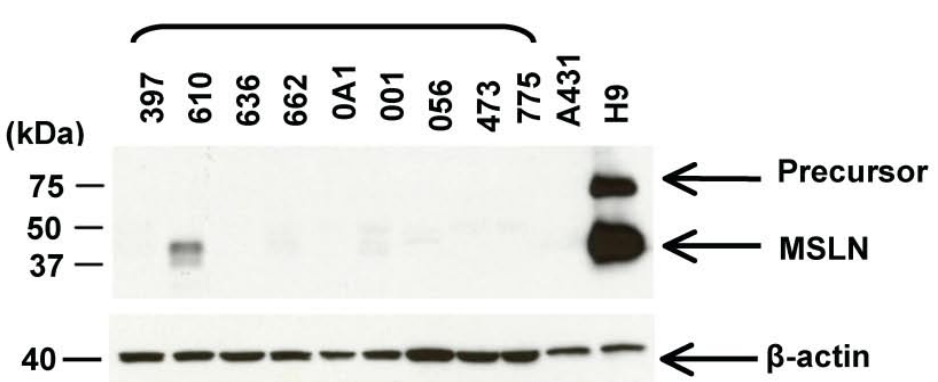

Figure 2. Characterization of mesothelin protein expression in liver cancers. (A) Immunoblotting analysis of mesothelin proteins in human liver cancer cell lines. Forty $\mu \mathrm{g}$ of whole cell lysate was loaded for each sample except A43I and H9 (only $2 \mu \mathrm{g}$ of total protein was loaded). (B) Immunoblotting analysis of mesothelin proteins in cancer specimens. Forty $\mu \mathrm{g}$ of whole cell lysate was loaded for each sample. OVCAR3 (a human ovarian cancer cell line) and H9 (A43I.MSLN+) were used as positive controls. A43I (MSLN-) was used as a negative control. MSLN: mesothelin ( 40 kDa); Precursor: 7I kDa. 
To investigate mesothelin as a potential surface target, we characterized cell surface expression of mesothelin in six CCA cell lines (HuCCT1, HuH-28, $\mathrm{KMBC}, \mathrm{KMCH}, \mathrm{Mz}-\mathrm{ChA}-1$ and OZ) by flow cytometry. As shown in Figure 3, four CCA cell lines (OZ, Mz-ChA-1, KMBC and KMCH) expressed mesothelin proteins. The levels of cell surface expression were consistent with the Western blotting intensities of the mature form of mesothelin whereas Mz-ChA-1 had relatively lower expression of mature or cell surface mesothelin as compared to $\mathrm{OZ}, \mathrm{KMBC}$ and $\mathrm{KMCH}$ (Fig. 2).

\section{Growth inhibition of immunotoxin SS IP on CCA cell lines}

To determine if mesothelin could function as a new therapeutic target in liver cancer, we tested SS1P, an anti-mesothelin recombinant immunotoxin [24].
The growth inhibition of immunotoxin SS1P was determined on six CCA cell lines by a WST cell proliferation assay (Fig. 4). LMB2 was used as a non-specific immuntoxin control. LMB2 is a recombinant immunotoxin that contains the $F v$ recognizing the $\alpha$-subunit of the IL-2 receptor (CD25) fused to a 38-kDa form of PE38. In the four CCA cancer cell lines with mesothelin expression on the cell surface (shown in Fig. 2), OZ, Mz-ChA-1, KMBC and KMCH, SS1P was very active with $\mathrm{IC}_{50}$ values ranging from 0.5 to $11 \mathrm{ng} / \mathrm{mL}$ (Fig. 4). In the HuCCT1 and HuH-28 cell lines with no or very low mesothelin expression, no significant growth inhibition $\left(\mathrm{IC}_{50}>1000 \mathrm{ng} / \mathrm{mL}\right.$ ) was observed. The high growth inhibition was comparable with the results of previous studies in mesothelioma and ovarian cancer [25].
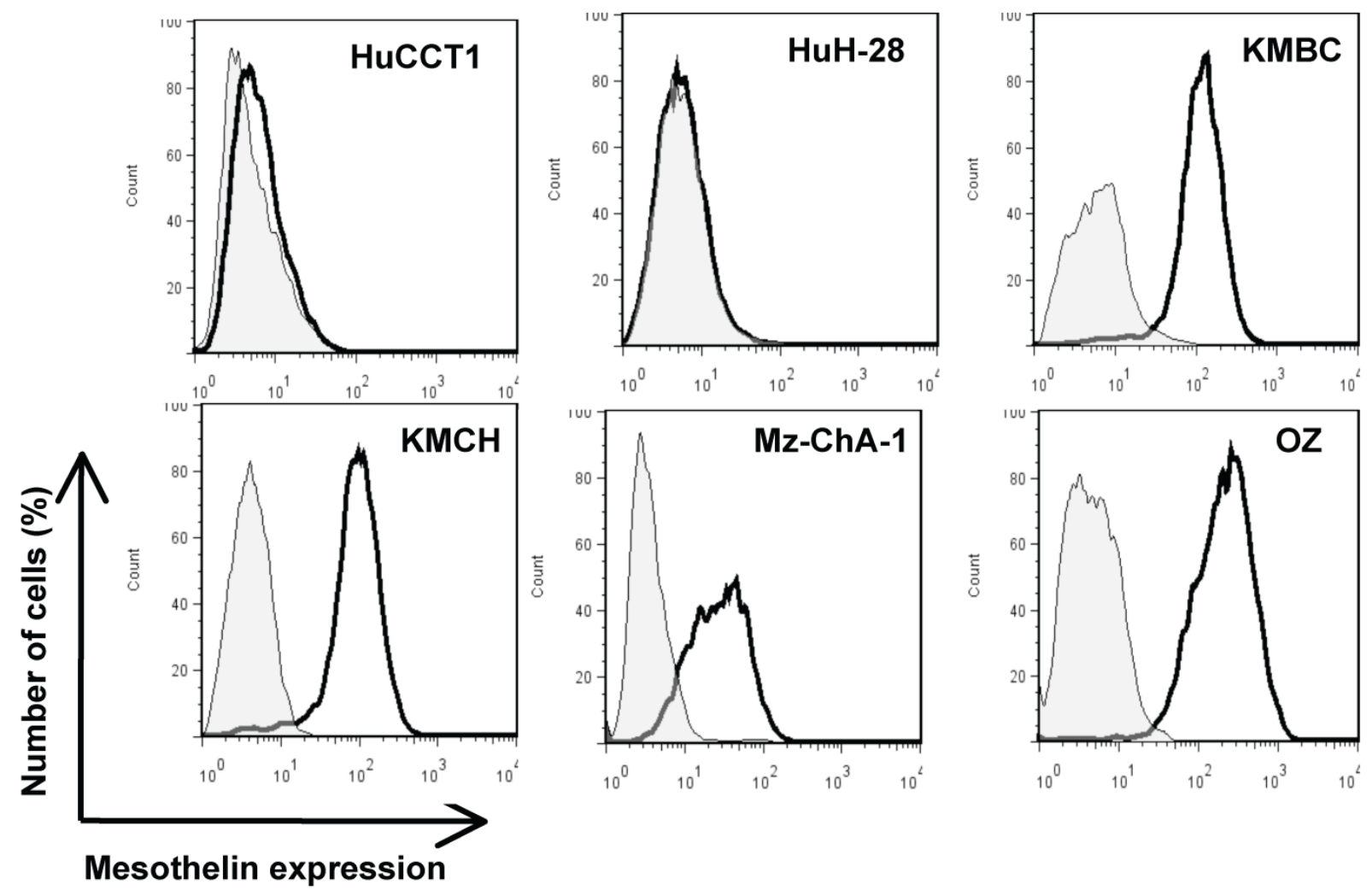

Figure 3. Flow cytometric analysis of mesothelin protein expression on CCA cells. Cells were probed with an anti-mesothelin $\mathrm{mAb}$ (solid dark line) or an irrelevant isotype mAb control (light gray shading). Each cell line and its GeoMean value: HuCCTI (6.2I), HuH-28 (5.54), KMBC (108.74), KMCH (84.63), Mz-ChA-I (25.19) and OZ (195.48). GeoMean of the isotype antibody control was about 5.0. 

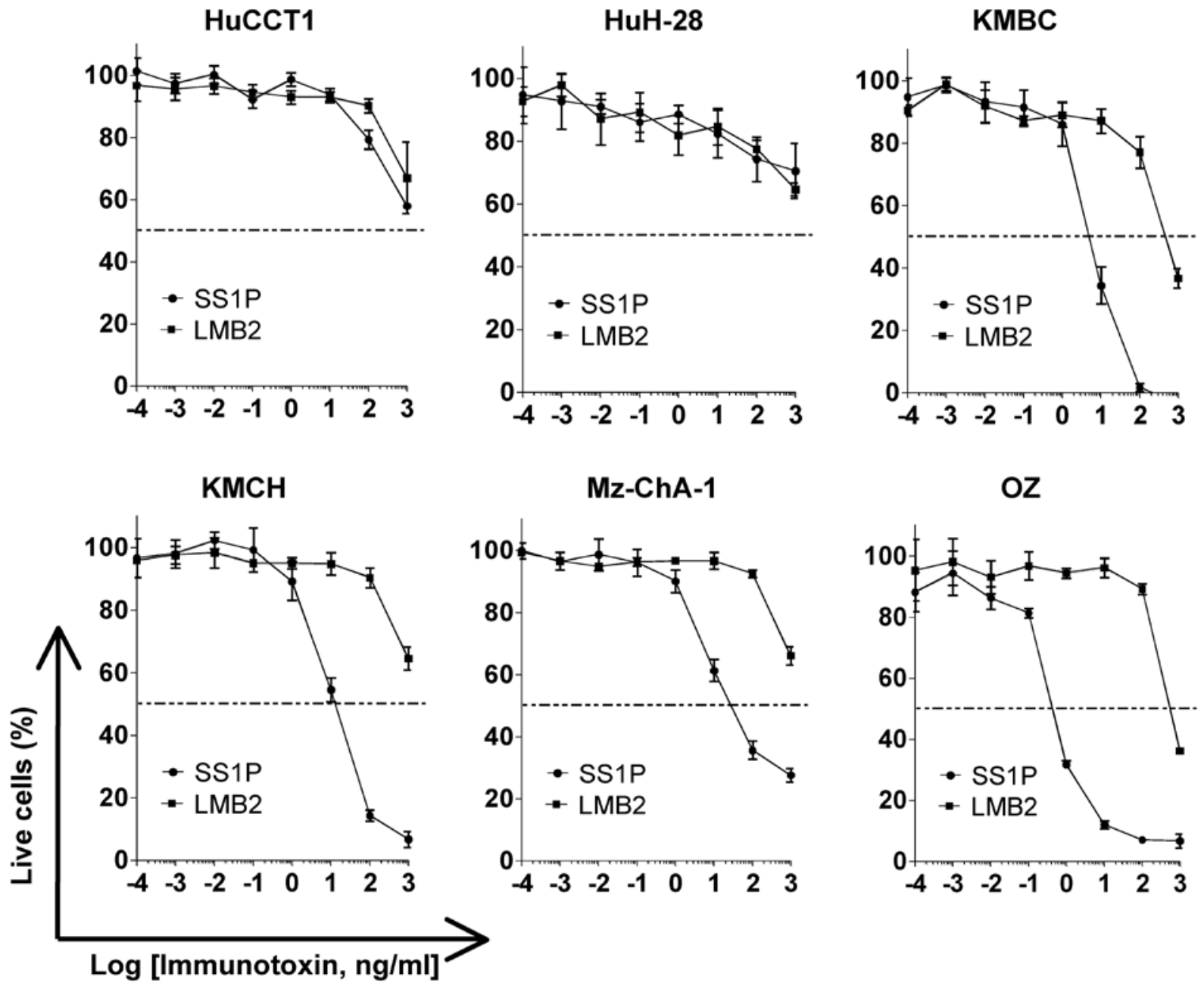

Figure 4. Inhibition of CCA cell proliferation by SSIP. Cancer cells (I0,000 per well) were incubated with various concentrations of SSIP. Cell proliferation was measured by a WST assay as described in Materials and Methods. Each point represents the mean of triplicate experiments and the error bars indicate the standard deviation. The dashed line indicates $50 \%$ inhibition of cell viability, which is halfway between the level of viability in the absence of toxin and that in the presence of $10 \mu \mathrm{g} / \mathrm{ml}$ of cycloheximide. Each cell line and its $\mathrm{IC}_{50}(\mathrm{ng} / \mathrm{mL}): \mathrm{HuCCTI}(>1000 \mathrm{ng} / \mathrm{ml}$ for both SSIP and LMB2), HuH-28 (>1000 ng/ml for both SSIP and LMB2), KMBC $(6.8 \mathrm{ng} / \mathrm{ml}$ for SSIP; $440.5 \mathrm{ng} / \mathrm{ml}$ for LMB2), KMCH (I0.7 ng/ml for SSIP; $>1000 \mathrm{ng} / \mathrm{ml}$ for LMB2), Mz-ChA-I (9.I ng/ml for SSIP; >1000 ng/ml for LMB2) and OZ (0.5 ng/ml for SSIP; > $1000 \mathrm{ng} / \mathrm{ml}$ for LMB2). LMB2, a PE immunotoxin control specific for CD25.

\section{Discussion}

There are two major findings in the present study. First, we found mesothelin was expressed in $33 \%$ of the surgically resected CCA specimens and also in 3 of 6 CCA cell lines but not in HCC specimens and cell lines. Second, we found that by treating the SS1P immunotoxin, the proliferations of CCA cell lines which expressed mesothelin were significantly inhibited.

Recently, Valle et al. reported a randomized, controlled, Phase III trial of 410 patients with locally advanced or metastatic CCA, gallbladder cancer, or ampullary cancer. In this trial, patients received either cisplatin followed by gemcitabine or gemcitabine alone for up to 24 weeks at 37 centers in the United Kingdom [31]. While the results are encouraging, the study strongly suggests that CCA remains very difficult to cure with current methods and molecular targeted therapy is urgently needed for this deadly disease.

Ordóñez found by IHC that $37 \%$ (7 of 19) of CCA showed mesothelin expression and none of HCC exhibited mesothelin expression [32]. Hassan et al. detected $100 \%$ (12 of 12) mesothelin expression in common bile duct adenocarcinoma using the 5B2 antibody [29]. Here, we showed by IHC that the expres- 
sion of mesothelin was present in 33\% of CCA. Normal liver tissues and HCC specimens showed no mesothelin expression. We also confirmed the IHC results by using the $\mathrm{mAb} \mathrm{MB}$. The results were found to be consistent although a relatively high non-specific background was found (data not shown). This difference may be due to the fact that 5B2 and MB bind two different epitopes in mesothelin. The binding of $\mathrm{MB}$ to mesothelin may be glycosylation dependent as previously suggested [27]. The present IHC study and previous reports [29,32], all with limited tumor specimens, have suggested a diagnostic potential of mesothelin in primary liver cancer, particularly CCA. An examination of additional patient studies from various human risk populations including specific clinicopathological features and prognostic values will provide further insight into mesothelin detection as a general diagnostic tool.

In the present study, we clearly demonstrate that SS1P is very active with $\mathrm{IC}_{50}$ values ranging from 0.5 to $11 \mathrm{ng} / \mathrm{mL}$ on mesothelin-expressing CCA cell lines. We have also shown that over $20 \%$ of CCA tumors have high mesothelin expression with levels comparable to the CCA cell line models evaluated in this work. SS1P should merit evaluation as a novel therapeutic agent for the treatment of primary liver cancer, particularly CCA. Further experiments in mouse models of cancer and human clinical trials could evaluate this possibility. A clinically relevant mouse tumor model to examine the efficacy of SS1P therapy is currently not available. Sirica and colleagues recently established a syngeneic rat model for CCA [33]. However, it is not a suitable model for SS1P due to the fact that the SS1 Fv is not cross-reactive with murine mesothelin. SS1P has been extensively evaluated in a nude mouse model in which an A431 human epithelial carcinoma cell line transfected with the human mesothelin cDNA forms solid tumors [25,34,35]. Furthermore, two Phase I clinical trials of SS1P have been completed in mesothelioma and ovarian cancer patients [25]. Based on Phase I clinical studies showing that SS1P is safe and has anti-tumor activity in heavily pretreated patients, Phase II studies of SS1P have recently begun. The ongoing clinical trials will help define the utility of mesothelin as a new target for cancer therapy. We believe that the findings in the present study demonstrate the potential for a new clinical trial of SS1P for the treatment of CCA. The investigation of such applications is a major focus of our current work.

\section{Acknowledgements}

We thank Dr. Ira Pastan (NCI) for helpful discussions, Dr. Alphonse E. Sirica (Virginia Commonwealth University) for critical reading of the manu- script, Drs. Mark Willingham (Wake Forest University), Raffit Hassan (NCI), Ilona Linnoila (NCI) and $\mathrm{Xu}$ Naizhen (NCI) for helpful advice on IHC preparation and analysis. We also thank the NIH Fellows Editorial Board for their review of the manuscript, Barbara Taylor (NCI Flow Cytometry Core) for technical assistance in flow cytometry, and Anna Mazzuca for editorial assistance. The content of this publication does not necessarily reflect the views or policies of the Department of Health and Human Services, nor does mention of trade names, commercial products, or organizations imply endorsement by the U.S. Government.

This work was supported in part by the Intramural Research Program of the National Institutes of Health, National Cancer Institute, Center for Cancer Research, by an Ovarian Cancer Research Fund Individual Investigator Award (M.H.), by a Mesothelioma Applied Research Foundation Grant in Honor of Craig Kozicki (M.H.), and by a key scientific and technological project from the Science and Technology Commission of Shanghai Municipality (074319104) (M.Q.).

\section{Conflict of Interest}

The authors have declared that no conflict of interest exists.

\section{References}

1. Spangenberg HC, Thimme R, Blum HE. Targeted therapy for hepatocellular carcinoma. Nat Rev Gastroenterol Hepatol. 2009; 6:423-32.

2. Shin HR, Oh JK, Masuyer E, Curado MP, Bouvard V, Fang YY, et al. Epidemiology of cholangiocarcinoma: an update focusing on risk factors. Cancer Sci. 2010; 101:579-85.

3. Sirica AE, Dumur CI, Campbell DJ, Almenara JA, Ogunwobi OO, Dewitt JL. Intrahepatic cholangiocarcinoma progression: prognostic factors and basic mechanisms. Clin Gastroenterol Hepatol. 2009; 7:S68-78.

4. Patel T. Increasing incidence and mortality of primary intrahepatic cholangiocarcinoma in the United States. Hepatology. 2001; 33:1353-7.

5. Shaib Y, El-Serag HB. The epidemiology of cholangiocarcinoma. Semin Liver Dis. 2004; 24:115-25.

6. Slattery JM, Sahani DV. What is the current state-of-the-art imaging for detection and staging of cholangiocarcinoma? Oncologist. 2006; 11:913-22.

7. Jarnagin WR, Shoup M. Surgical management of cholangiocarcinoma. Semin Liver Dis. 2004; 24:189-99.

8. Ben-Menachem T. Risk factors for cholangiocarcinoma. Eur J Gastroenterol Hepatol. 2007; 19:615-7.

9. Chang K, Pastan I, Willingham MC. Isolation and characterization of a monoclonal antibody, K1, reactive with ovarian cancers and normal mesothelium. Int J Cancer. 1992; 50:373-81.

10. Argani P, Iacobuzio-Donahue C, Ryu B, Rosty C, Goggins M, Wilentz RE, et al. Mesothelin is overexpressed in the vast majority of ductal adenocarcinomas of the pancreas: identification of a new pancreatic cancer marker by serial analysis of gene expression (SAGE). Clin Cancer Res. 2001; 7:3862-8. 
11. Ho M, Bera TK, Willingham MC, Onda M, Hassan R, FitzGerald $\mathrm{D}$, et al. Mesothelin expression in human lung cancer. Clin Cancer Res. 2007; 13:1571-5.

12. Chang K, Pastan I. Molecular cloning of mesothelin, a differentiation antigen present on mesothelium, mesotheliomas, and ovarian cancers. Proc Natl Acad Sci USA. 1996; 93:136-40.

13. Rump A, Morikawa Y, Tanaka M, Minami S, Umesaki N, Takeuchi $\mathrm{M}$, et al. Binding of ovarian cancer antigen CA125/MUC16 to mesothelin mediates cell adhesion. J Biol Chem. 2004; 279:9190-8.

14. Gubbels JA, Belisle J, Onda M, Rancourt C, Migneault M, Ho M, et al. Mesothelin-MUC16 binding is a high affinity, N-glycan dependent interaction that facilitates peritoneal metastasis of ovarian tumors. Mol Cancer. 2006; 5:50-65.

15. Kaneko O, Gong L, Zhang J, Hansen JK, Hassan R, Lee B, et al. A binding domain on mesothelin for CA125/MUC16. J Biol Chem. 2009; 284:3739-49.

16. Lopez JB, Balasegaram M, Timor J, Thambyrajah V. Comparison of alpha-fetoprotein with some other tumour markers in Malaysians with hepatocellular carcinoma. Malays J Pathol. 1997; 19:53-8

17. Su WC, Chan KK, Lin XZ, Lin PW, Chow NH, Shin JS, et al. A clinical study of 130 patients with biliary tract cancers and periampullary tumors. Oncology. 1996; 53:488-93.

18. Ho M, Onda M, Wang QC, Hassan R, Pastan I, Lively MO. Mesothelin is shed from tumor cells. Cancer Epidemiol Biomarkers Prev. 2006; 15:1751.

19. Scholler N, Fu N, Yang Y, Ye Z, Goodmn GE, Hellström KE, et al. Soluble member(s) of the mesothelin/megakaryocyte potentiating factor family are detectable in sera from patients with ovarian carcinoma. Proc Natl Acad Sci USA. 1999; 96:11531-6.

20. Robinson BW, Creaney J, Lake R, Nowak A, Musk AW, de Kierk N, et al. Soluble mesothelin-related protein--a blood test for mesothelioma. Lung Cancer. 2005; 49(Suppl 1):S109-11.

21. Hassan R, Remaley AT, Sampson ML, Zhang J, Cox DD, Pingpank J, et al. Detection and quantitation of serum mesothelin, a tumor marker for patients with mesothelioma and ovarian cancer. Clin Cancer Res. 2006; 12:447-53.

22. Hellstrom I, Raycraft J, Kanan S, Sardesai NY, Verch T, Yang Y, et al. Mesothelin variant 1 is released from tumor cells as a diagnostic marker. Cancer Epidemiol Biomarkers Prev. 2006; 15:1014-20

23. Ho M, Hassan R, Zhang J, Wang QC, Onda M, Bera T, et al. Humoral immune response to mesothelin in mesothelioma and ovarian cancer patients. Clin Cancer Res. 2005; 11:3814-20.

24. Pastan I, Hassan R, Fitzgerald DJ, Kreitman RJ. Immunotoxin therapy of cancer. Nat Rev Cancer. 2006; 6:559-65.

25. Hassan R, Ho M. Mesothelin targeted cancer immunotherapy. Eur J Cancer. 2008; 44:46-53.

26. Hassan R, Ebel W, Routhier EL, Patel R, Kline JB, Zhang J, et al. Preclinical evaluation of MORAb-009, a chimeric antibody targeting tumor-associated mesothelin. Cancer Immun. 2007; 7:20.

27. Onda M, Willingham M, Nagata S, Bera TK, Beers R, Ho M, et al. New monoclonal antibodies to mesothelin useful for immunohistochemistry, fluorescence-activated cell sorting, Western blotting, and ELISA. Clin Cancer Res. 2005; 11:5840-6.

28. McShane LM, Altma DG, Sauerbrei W, Taube SE, Gion M, Clark GM. Reporting recommendations for tumor marker prognostic studies (REMARK). Statistics Subcommittee of the NCI-EORTC Working Group on Cancer Diagnostics. J Natl Cancer Inst. 2005; 97:1180-4.

29. Hassan R, Laszik ZG, Lerner M, Raffeld M, Postier R, Brackett $D$. Mesothelin is overexpressed in pancreaticobiliary adenocarcinomas but not in normal pancreas and chronic pancreatitis. Am J Clin Pathol. 2005; 124:838-45.
30. Heffelfinger SC, Hawkins HH, Barrish J, Taylor L, Darlington GJ. SK HEP-1: a human cell line of endothelial origin. In Vitro Cell Dev Biol. 1992; 28:136-42.

31. Valle J, Wasan H, Palmer DH, Cunningham D, Anthoney A, Maraveyas A, et al. Cisplatin plus gemcitabine versus gemcitabine for biliary tract cancer. N Engl J Med. 2010; 362:1273-81.

32. Ordóñez NG. Application of mesothelin immunostaining in tumor diagnosis. Am J Surg Pathol. 2003; 27:1418-28.

33. Sirica AE, Zhang Z, Lai GH, Asano T, Shen XN, Ward DJ, et al. A novel "patient-like" model of cholangiocarcinoma progression based on bile duct inoculation of tumorigenic rat cholangiocyte cell lines. Hepatology. 2008; 47:1178-90.

34. Zhang Y, Xiang L, Hassan R, Paik CH, Carrasquillo JA, Jang BS, et al. Synergistic antitumor activity of taxol and immunotoxin SS1P in tumor-bearing mice. Clin Cancer Res. 2006; 12:4695-701.

35. Zhang Y, Hansen JK, Xiang L, Kawa S, Onda M, Ho M, et al. A flow cytometry method to quantitate internalized immunotoxins shows that taxol synergistically increases cellular immunotoxins uptake. Cancer Res. 2010; 70:1082-9. 